\title{
INFLUENCE OF LAY PLAN SOLUTION IN FABRIC EFFICIENCY AND CONSUME IN CUTTING SECTION
}

\author{
Elmira Dumishllari, Genti Guxho
}

Department of Textile and Fashion, Polytechnic University of Tirana, Albania

edymishllari@fim.edu.al

\begin{abstract}
:
One cutting order may require several lies and markers to achieve optimal efficiency and selection of right Lay Plan is one of the major challenges in cutting section, in a way to lead in fabric economy and reduced costs. The main purpose of this paper is to determine the best solution of lay plan and its influence, in the cutting room, starting from the analysis of the lay indicators calculations. We have studied an order with 200 jackets, with specific sizes. Markers are made by Gemini CAD (Gemini Cut Plan and Gemini Nest Expert). From Gemini CAD, we have taken different solutions of lay plans and chose the three best of them (lay plans that have a smaller number of lies, plies etc.), and then, for three best solutions are calculated lay indicators. From this calculation, we have determined the best solution of lay plan. The order to study has 5 different fabrics, so the calculations are made for each of them, and in the end are combined, in a way to have conclusions for an order in general. Best solution of lay plans combination, resulted one which had a better weighted efficiency and a low lay consumption.
\end{abstract}

\section{Keywords:}

Lay plan, marker, fabric, efficiency, plies.

\section{Introduction}

Cut Order Planning is deciding the combination of markers, and lays for a particular order. This activity is also termed as "lay plan", "cut plan", "lay lot plan", etc. [10].

A marker is a diagram of a precise arrangement of pattern pieces for a specific style and the sizes to be cut from a single spread [14], or marker, is a thin paper which contains all necessary pattern pieces for all sizes for a particular style of garments in such a way that, fabric wastage would be least [2]. A lay is a stack of fabric plies that have been prepared for cutting. A lay planning is the basis of managing cutting room labor and table space [14].

A major activity of the cut - plan is marker making that is the process of determining the most efficient layout of the pattern pieces for a specific style, fabric, and the distribution of sizes [14]. Marker making is a critical step in the manufacturing process [8]. Fabric loss in cutting room is mainly caused by two operations- marker making and fabric spreading [3]. Traditional manufacturers manage the process of marker making in-house. By retaining strict control over this critical step, they keep the fabric consumption as low as possible. Controlling this step also permits them to ensure that the issues that affect quality will be given proper attention. Depending on the relative efficiency of each marker produced, the company may save or waste money. The methods chosen for these steps have a direct effect on the quality and cost of the finished garment [12], [7].

The marker making can be broken down into following two sections: Marker Planning and Marker Production [12], [4].

Marker Planning is the placement of pattern pieces to meet the technical requirements and needs of the material economy [4]. Marker Production may include drawing of marker plan directly on the fabric, drawing it onto a paper marker by pen or automatic plotter, or, where the cutting method allows it, recording pattern piece information on the paper marker or on the fabric without actually drawing pattern lines on it [12], [4]. A CAD program is useful because it automatically calculates the yardage needed and indicates how many layers of fabric need to be spread in order to complete the desired number of products. Each fabric layer is called a "ply" [5]. The use of computer involves big investment, but at the same time advantage is that it saves time, gives more opportunity, options, and accuracy [1]. CAD systems are extensively used during the preproduction phase, which is labor-intensive [11].

The greatest attention is always given for the marker planning. Any reduction in the amount of the cloth used per garment leads to increased profit [12]. The minimization of fabric wastage is crucial to the reduction of production costs [15]. Marker planning is always a very open \& creative process. Marker planning is a highly skilled activity and different people have different attitudes for this kind of work [8]. Computerized marker making can assist but rarely replace skilled people. General procedure for marker planning include: The large pattern pieces have to be positioned first and then fitting the smaller pieces into the gaps, Most of the pattern pieces are irregular and often tapered, the skill lies in discovering those edges which fit together most neatly, and placing side by side across the marker those pieces that fill the width most nearly, the marker planner has to try a number of pattern placements, selecting the one that gives the shortest marker [12].

Raw materials management is one of the three most important areas where firms are focusing their cost reduction efforts [9]. 
This study was carried out in an apparel production companies, operating CMT, at cutting section, to identify the best solution of lay plan in a way to save fabric and consequently money. These companies produce different garments for mass production. Data were taken in one of these companies.

\section{Experimental}

\subsection{Materials}

The study is focused on realization of several lay plans, for a given order, in cutting section in way to achieve optimal efficiency. We know that markers may be made by manually tracing master patterns onto fabric or paper or by manipulating and plotting computerized pattern images [14]. In this study, we used the second method of marking, and the softwares that the company uses are Gemini CAD (Gemini Cut Plan and Gemini Nest Expert). With Gemini Cut Plan (GCP), we can be able to plan the size combinations on markers and to plan the lays to obtain the required quantities, calculate the input and efficiency, set and print the order report, print the markers on a plotter, export the markers for the automatic cutting machine and save and manage data in files on the computer hard disk or in databases for cutting plans. The Gemini Nest Expert application is used to optimize the markers [6].

We have studied an order with 200 jackets of sizes 38-40-42-4446-48-50-52-54, with respectively 15, 20, 30, 30, 35, 25, 20, 15, 10 pieces. The jacket is for casual use, deep blue color, with two front pockets and consists of these main parts: front, back, shoulder, two front pockets, two pocket's flaps, collar, etc. Besides the main material (fabric), a part of the jacket has a polyester textile with boxes (white, blue and red colors), lining and a white fabric in the embroidery that is in the shoulder. In this article we will present, mainly, the calculated results of main fabric. And, the calculations of four other fabrics will appear in tables.

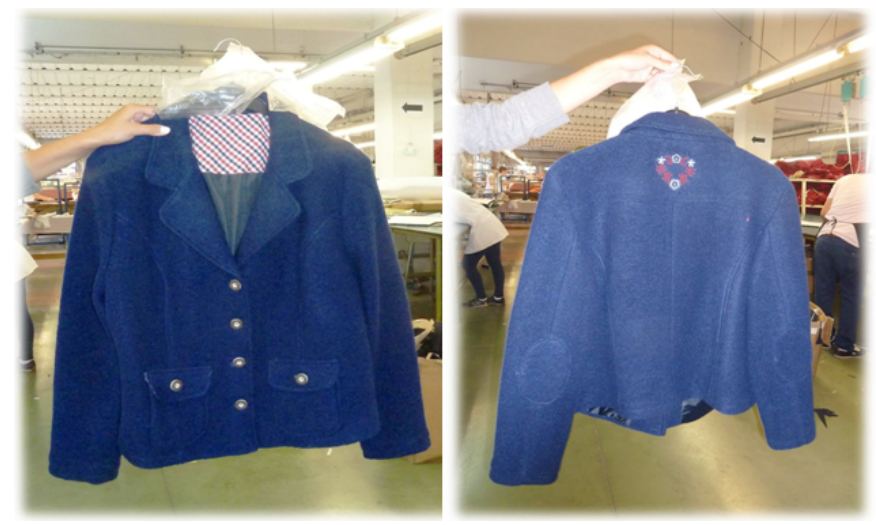

Figure 1. Jacket's the front (face) and back view

\subsection{Methods}

The method used in this study is based in:

1. Plan and realize the lays to obtain the required quantities according to the given order and plan and realize the size combinations of markers (in a way to have the best results of efficiency).
2. Analyze of calculations (lay indicators) for the main fabric, and four other non - main fabrics, for the three best solutions, considered suitable in accordance with our requirements and indicator's calculation for the order in general

\section{Discussion of results.}

\section{Results and discussion}

One cutting order may require several markers to achieve optimal efficiency. Marker efficiency is determined by fabric utilization, the percentage of the total fabric that is actually used in garment parts. The area not used in garment parts is waste. Marker efficiency depends on how tightly the pattern pieces fit together within the marker. The total surface area of the pattern pieces is compared to the total area of the marker to calculate the percentage of fabric that is used [14]. In our study this is determined automatically by the marker-making software Gemini CAD. The three parameters of interest for having the highest efficiency are: the smaller length of markers, the smaller number of markers, the smaller length of the fabric for a marker. A higher efficiency marker results in better savings [10]. Marker efficiency, a subset of Cut Order Planning, is much hyped, as it is easily quantifiable in terms of percentage of fabric consumption. There are many facets of cut Order Planning, and reducing fabric consumption is just one of them [13]. Fabric is the core of apparel manufacturing [3].

We need to make a selection of sizes to be placed together in a marker for the purpose of textile economy. The way how the lay plan will be done, is decided by the layer man based on work experience, in order to achieve a higher efficiency. Different lay plan give a different percentage of weighted efficiency. We will see three solutions of lay plan and will choose the most efficient of them.

\subsection{The first solution of lay plan (main fabric)}

As we see from the table 1 , will be done 4 lays. The first lay, with sizes 42-44-46-48, will be done with 30 plies (layers). The second lay, with sizes 38-40-50-52, will be done with 15 plies and the third lay with sizes 40-46-50-54 will be done with 5 plies. In the last lay, the forth, we have only 54 size, with 5 plies.

Table 1. The first lay plan of main fabric

\begin{tabular}{|c|c|c|c|c|c|c|c|c|c|c|c|c|c|}
\hline \multirow{3}{*}{$\begin{array}{l}\text { New fabri } \\
\text { Layers } \\
\end{array}$} & \multirow{3}{*}{\multicolumn{2}{|c|}{$\frac{\mid}{\text { ic } \mid}$}} & \multirow{3}{*}{ Qty } & \multirow{2}{*}{\multicolumn{9}{|c|}{12354 BA KII59 - Defaut }} & \multirow[b]{3}{*}{ Marker } \\
\hline & & & & & & & & & & & & & \\
\hline & & & & 38 & 40 & 42 & 44 & 46 & 48 & 50 & 52 & 54 & \\
\hline & & & & 15 & 20 & 30 & 30 & 35 & 25 & 20 & 15 & 10 & \\
\hline \multirow[t]{2}{*}{30} & Simple & $>$ & 4 & 0 & 0 & 1 & 1 & 1 & 1 & 0 & 0 & 0 & $42 ; 44 ; 46 ; 48 ;$ \\
\hline & & & & 15 & 20 & 0 & 0 & 5 & +5 & 20 & 15 & 10 & \\
\hline \multirow[t]{2}{*}{15} & Simple & $>$ & 4 & 1 & 1 & 0 & 0 & 0 & 0 & 1 & 1 & 0 & $38 ; 40 ; 50 ; 52 ;$ \\
\hline & & & & 0 & 5 & 0 & 0 & 5 & +5 & 5 & 0 & 10 & \\
\hline \multirow[t]{2}{*}{5} & Smple & $>$ & 4 & 0 & 1 & 0 & 0 & 1 & 0 & 1 & 0 & 1 & $40 ; 46 ; 50 ; 54 ;$ \\
\hline & & & & 0 & 0 & 0 & 0 & 0 & +5 & 0 & 0 & 5 & \\
\hline \multirow[t]{2}{*}{5} & Simple & $>$ & 1 & 0 & 0 & 0 & 0 & 0 & 0 & 0 & 0 & I & 54; \\
\hline & & & & 0 & 0 & 0 & 0 & 0 & +5 & 0 & 0 & 0 & \\
\hline
\end{tabular}

What is the logic of the lay plan? 
The first lay with 30 layers (The first marker). At the beginning are taken into account sizes which will produce a larger number of products, compared with other sizes. In a marker we have a combination of sizes with each other, in order to economize textile. It is understood that from a textile ply we get a product for each of the sizes contained in the marker. In our case sizes 42-44-46-48 have more products to be cast in manufacturing. So if we decide to make the first lay with 30 plies, will take 30 jackets of sizes 42 and 44 that we need. Will remain unrealized 5 jackets of size 46 , but they will be realized in a lay with 5 plies. For the size 48 we need 25 jackets, but including this size in a lay with 30 plies we receive 5 jackets more than asked, but not to exceed the amount of textile we have available to use. This can be accomplished when it comes to a small number of products, otherwise remaining textile is stored for use in any other similar order, or simply stored.

So at the first marker, of the first lay plan, we included 4 sizes and in the table 1 is named quantity Qty. The sizes appear automatically on "Marker" and are: 42, 44, 46, 48.

- The second lay with 15 plies (The second marker). From the first lay remain to be involved in marker 15 jackets of size 38 , 20 jackets of size 40, 5 jackets of size 46,20 jackets of size 50,15 jackets of size 52 and 10 jackets of size 54 . We have chosen to do a lay with 15 plies, including sizes 38-40-50-52.

Why we did not choose to do a lay with 20 plies?

If we had decided to do a laying with 20 plies, would have involved only 40 and 50 sizes, of which are required 20 jackets and we would set aside 38 and 52 sizes. As we see with 15 plies is achieved laying the more sizes and that we care. In this way we finished with size 38 and the remaining 5 jackets of size 40 will be put in a lay with 5 plies. The same goes for the 50 size. With the size 52 have finished, while 54 size will remain for another lay.

- The third lay with 5 plies (The third marker). The current state of the remaining sizes, not included in the first and second lay is: 5 jackets from each of three sizes $(40,46,50)$ and 10 jackets of 54 size. If we had decided to do a lay with 10 plies, would have did not include three sizes, while the 5 plies include four measures: 40-46-50-54.

- For the size 54 , have not been included in the marker 5 jackets for which will make a last lay with 5 plies.

\subsection{The second solution of lay plan (main fabric)}

Table 2. The second solution of lay plan of main fabric

\begin{tabular}{|c|c|c|c|c|c|c|c|c|c|c|c|c|c|}
\hline$=$ & 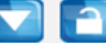 & & 항의 & Solutic & & & & & & & & 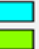 & $\begin{array}{l}\text { - manual } \\
\text { - automa }\end{array}$ \\
\hline \multicolumn{14}{|c|}{ New fabric } \\
\hline & & & L & \multicolumn{8}{|c|}{ |12354_BA_KLI59 - Default } & & \\
\hline \multirow[t]{2}{*}{ Layers } & \multicolumn{2}{|l|}{ Layer type } & Qty & 38 & 40 & 42 & 44 & 46 & 48 & & 50 & 52 & 54 \\
\hline & & & & 15 & 20 & 30 & 30 & 35 & 25 & & 20 & 15 & 10 \\
\hline \multirow[t]{2}{*}{20} & Simple & $>$ & 4 & 0 & 1 & 1 & 0 & 0 & 1 & & 1 & 0 & 0 \\
\hline & & & & 15 & 0 & 10 & 30 & 35 & 5 & & & 15 & 10 \\
\hline \multirow[t]{2}{*}{15} & Simple & $>$ & 4 & 1 & 0 & 0 & 1 & 1 & 0 & & & 1 & 0 \\
\hline & & & & 0 & 0 & 10 & 15 & 20 & 5 & & & 0 & 10 \\
\hline \multirow[t]{2}{*}{10} & Simple & $>$ & 4 & 0 & 0 & 1 & 1 & 1 & 0 & & & 0 & 1 \\
\hline & & & & 0 & 0 & 0 & 5 & 10 & 5 & & & 0 & 0 \\
\hline 5 & Simple & $>$ & 4 & 0 & 0 & 0 & 1 & 2 & 1) & & D & 0 & 0 \\
\hline
\end{tabular}

- The first lay with 20 plies (The first marker). We have mentioned above that for a better economy of fabric, will combine small sizes with the big sizes, thus in the first lay with 20 plies will put sizes $40-42-48-50$. Given that, from a ply we receive a product for each of the sizes selected, for the 40 size, we realized 20 jackets, as well as measure 50 . From 42 size remain unrealized 10 pieces (from 30 that is the order), and by size 48 remain 5 jackets.

- The second lay with 15 plies (The second marker). In this lay will include the sizes 38-44-46-52. From the measures 38 and 52 remained unrealized 15 jackets (for each of them), so they will be included in this lay with 15 plies. Remain not included 15 pieces by the size 44 and 20 by the 46 size.

- The third lay with 10 plies (The third marker). In the third lay we have put the sizes 42-44-46-54. We have finished with the sizes 42 and 54 , while from the 44 size remain 5 pieces and from 46 size remain 10 pieces.

- The fourth lay with 5 plies (The fourth marker). The sizes that are left are: 44-46-48. We need 5 pieces for 44 size, 10 pieces per size 46 and 5 pieces per size 48 . Given that all markers have 4 sizes, the size 46 can be placed twice, so will directly receive 10 jackets. So in the last marker will be the sizes $44-$ $46(\times 2)-48$.

\subsection{The third solution of lay plan (main fabric)}

We have analyzed a a third solution of lay plan, to understand which of three is more efficient. In this lay plan will be realized again 4 lays: the first lay with 15 plies contains sizes 38-46-4852 , the second lay with 20 plies contains sizes 40-44-46-50, the third lay by 10 plies contains sizes $42-44-48-54$ and the fourth with 5 plies contains 4 times the size 42 .

Table 3. The third solution of lay plan (for main fabric)

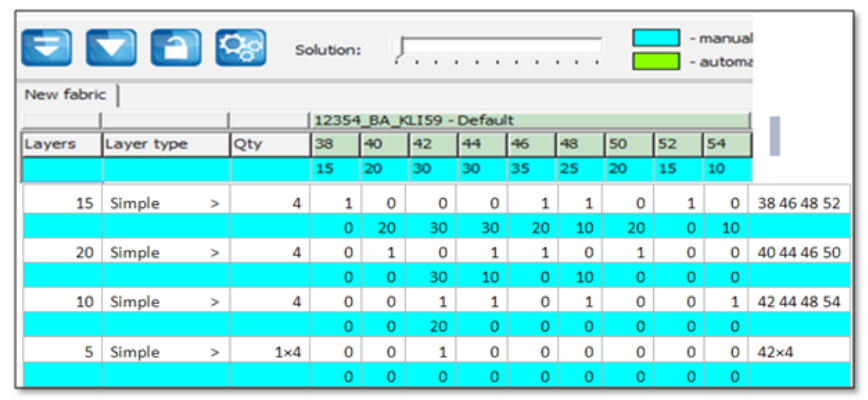

\subsection{Comparison of three solutions of lay plan, for five fabrics and the order in general.}

Let's prove that the right lay plan saves fabric. To estimate the quality of markers across the order, weighted efficiency metric should be used. This tells us the efficiency of markers over the whole order, weighted according to its number of plies [10]. First, we will calculate the weighted efficiency of the three solutions of lay plan, for the main fabric. From the Gemini CAD we have the marker efficiency and the length for each lay. In the length of a marker we should add the tolerance of $5 \mathrm{~cm}$ $(2.5 \mathrm{~cm}$ on each side). 
AUTEX Research Journal, Vol. 16, No 4, December 2016, DOI: 10.1515/aut-2015-0055 @ AUTEX

Table 4. Efficiency and length for three solutions of lay plan, generated from Gemini CAD

\begin{tabular}{|c|c|c|c|c|c|c|c|c|}
\hline Lay Plan1 & $\begin{array}{c}\text { Efficiency } \\
{[\%]}\end{array}$ & $\begin{array}{c}\text { Length } \\
{[\mathbf{m}]}\end{array}$ & Lay Plan2 & $\begin{array}{c}\text { Efficiency } \\
{[\%]}\end{array}$ & $\begin{array}{c}\text { Length } \\
{[\mathbf{m}]}\end{array}$ & Lay Plan3 & $\begin{array}{c}\text { Efficiency } \\
{[\%]}\end{array}$ & $\begin{array}{c}\text { Length } \\
{[\mathbf{m}]}\end{array}$ \\
\hline Lay 1 & 80.4 & 5.422 & Lay 1 & 79.2 & 5.518 & Lay 1 & 83.20 & 5.349 \\
\hline Lay 2 & 80.73 & 5.434 & Lay 2 & 78.12 & 5.6 & Lay 2 & 83.19 & 5.251 \\
\hline Lay 3 & 80.35 & 5.664 & Lay 3 & 80.11 & 5.581 & Lay 3 & 83.27 & 5.423 \\
\hline Lay 4 & 74.32 & 1.73 & Lay 4 & 78.26 & 5.657 & Lay 4 & 84.06 & 4.925 \\
\hline
\end{tabular}

Weighted efficiency $1=\frac{\sum(\text { Efficiency X Plies })}{\text { Total plies }}=\frac{(80.4 \times 120)+(80.73 \times 60)+(80.35 \times 20)+(74.32 \times 5)}{205}$
$=80.34 \%$

Weighted efficiency $2=\frac{\sum(\text { Efficiency X Plies })}{\text { Total plies }}=\frac{(79.2 \times 80)+(78.12 \times 60)+(80.11 \times 40)+(78.26 \times 20)}{200}$ $=78.96 \%$

Weighted efficiency3 $=\frac{\sum(\text { Efficiency X Plies })}{\text { Total plies }}=\frac{(83.2 \times 60)+(83.19 x 80)+(83.27 \times 40)+(84.06 x 20)}{200}$ $=83.29 \%$

We see that the third solution has a higher weighted efficiency than the two other lay plans.

Let's estimate now the lay consumption for the three lay plans. Lay consumption is an indicator of overall consumption of the order and it takes into account the influence of all the markers over the order in terms of plies [10].

$$
\begin{aligned}
& \text { Lay consumption } 1=\frac{\sum(\text { Marker length } X \text { Plies })}{\text { Total pieces cut }}=\frac{(5.422 \times 30)+(5.434 \times 15)+(5.664 \times 5)+(1.730 \times 5)}{205} \\
& =1.371 \mathrm{~m} \text {. } \\
& \text { Lay consumption } 2=\frac{\sum(\text { Marker length X Plies })}{\text { Total pieces cut }}=\frac{(5.518 \times 20)+(5.600 \times 15)+(5.581 \times 10)+(5.657 \times 5)}{200} \\
& =1.392 \mathrm{~m} \\
& \text { Lay consumption } 3=\frac{\sum(\text { Marker length } X \text { Plies })}{\text { Total pieces cut }}=\frac{(5.349 \times 15)+(5.251 \times 20)+(5.423 \times 10)+(4.925 \times 5)}{200} \\
& =1.320 \text { metres. }
\end{aligned}
$$

The usage of fabric will be based on lay consumption. This number is necessary for calculating fabric requirements. The order quantity is 200 pieces ( 205 in the first solution), so for the three cases the fabric consumption will be:

Total consumption $1=$ Lay consumption $1 X$ Total pieces cut $=1.371 X 205=281.055$ metres.

Total consumption $2=$ Lay consumption $2 X$ Total pieces cut $=1.392 X 200=278.4$ meters. Total consumption $3=$ Lay consumption 3 Total pieces cut $=1.320 X 200=264$ metres.

Table 5. Some parameters of three solutions

\begin{tabular}{|c|c|c|c|c|c|}
\hline & Weighted effic. & Lay consum. & No. markers & No. plies & Total consumption \\
\hline Solution 1 & 80.34 & 1.371 & 4 & 55 & 281.055 \\
\hline Solution 2 & 78.96 & 1.392 & 4 & 50 & 278.4 \\
\hline Solution 3 & 83.29 & 1.320 & 4 & 50 & 264 \\
\hline
\end{tabular}

We see that the third solution of lay plan has a better fabric consumption; $6.46 \%$ lower that the first solution and $5.45 \%$ lower that 
the second solution. Number of markers are same for three solutions, this means that the cost of marker making is same. Number of plies is same for second and third solution and in the firs is higher and this is an indicator of high spreading and fabric cost. From the total consumption, we see that the third solution reduces fabric cost, and that's why is the best. Based in the higher number of plies (higher that the ideal plies [10] that is calculated like number of total pieces cut / quantity per lay, ie 200/4=50) the first solution is the worst.

All this calculation was made for the main fabric.

The same calculations were made for four other fabrics, and results are presented in the table below.

Then, is made the combination of the first solutions of five fabrics, the second solutions of five fabrics and the third solutions of them. Are calculated the lay indicators of total order and presented in the table below. Also, in the end is made a combination of the best solutions for each fabric and calculated order's indicators.

From the results obtained, from the four solutions (including combined solution), was observed that third and fourth combinations are the best solutions because they have a better total consumption of fabric (lower than in other cases), a smaller number of plies. The first combination is not a good choice, as it has violated the ideal number of plies for each fabric and this number is higher than in the other combinations.
The third and combined solutions can be considered successful at the same time.

For the reason that the third combinations have the same laying strategy, are considered more suitable (from the point of time economy).

Main fabric and liner have significant difference in consumption by a lay plan solution to another.

\section{Conclusions}

From the analyses made for three solutions of the lay plan (for main fabric) we conclude that the third solution of lay plan has a better fabric consume; lower that the first and second solution. Number of markers are same for three solutions, this means that the cost of marker making is same. Number of plies is same for second and third solution and in the firs this number is higher and this is an indicator of high spreading and fabric cost. From the total fabric consume, we see that the third solution reduces fabric cost, and that's why is the best. Based in the higher number of plies the first solution is the worst.

From the results obtained, from the four solutions (including combined solution), was observed that third and fourth combinations are the best solutions because they have a better total consumption of fabric (lower than in other cases), a smaller number of plies.

Table 6. Some parameters of three solutions of lay plan for non - main fabric

\begin{tabular}{|c|c|c|c|c|c|c|}
\hline Fabrics & & $\begin{array}{c}\text { Weighted } \\
\text { Effic. [\%] }\end{array}$ & $\begin{array}{c}\text { Lay Consum } \\
{[\mathrm{m}]}\end{array}$ & No. markers & No. plies & $\begin{array}{c}\text { Tot. } \\
\text { Consump. [m] }\end{array}$ \\
\hline $\begin{array}{c}\text { Fabric with } \\
\text { boxes }\end{array}$ & 69.50 & 0.099 & 2 & 20 \\
\hline \multirow{2}{*}{ Lining } & Solution 1 & 63.29 & 0.275 & 4 & 55 & 56.375 \\
\cline { 2 - 7 } & Solution 2 & 61.98 & 0.277 & 4 & 50 & 55.40 \\
\cline { 2 - 7 } & Solution 3 & 61.729 & 0.2779 & 4 & 1 & 55.58 \\
\hline \multirow{2}{*}{$\begin{array}{c}\text { Embroidery's } \\
\text { fabric }\end{array}$} & Solution 1 & 81.77 & 0.912 & 3 & 50 & 186.96 \\
\hline \multirow{2}{*}{ Liner } & Solution 2 & 80.42 & 0.883 & 4 & 50 & 176.6 \\
\cline { 2 - 7 } & Solution 3 & 80.04 & 0.860 & 4 & 50 & 172 \\
\hline
\end{tabular}

Table 7. Some parameters of four combinations of lay plans of an order

\begin{tabular}{|c|c|c|c|c|c|c|}
\hline & $\begin{array}{c}\text { Recommended. } \\
\text { Length [m] }\end{array}$ & No. markers & No. lays & $\begin{array}{c}\text { No. } \\
\text { Plies }\end{array}$ & $\begin{array}{c}\text { Tot. Consump. } \\
{[\mathrm{m}]}\end{array}$ & $\begin{array}{c}\text { Weighted effic. } \\
{[\%]}\end{array}$ \\
\hline 1 & 6 & 14 & 14 & 181 & 546.74 & 73.61 \\
\hline 2 & 6 & 15 & 15 & 171 & 532.7 & 72.59 \\
\hline 3 & 6 & 15 & 15 & 171 & 513.88 & 73.5 \\
\hline 4 & 6 & 15 & 15 & 171 & 513.7 & 73.56 \\
\hline
\end{tabular}


The third and combined solutions can be considered successful at the same time.

For the reason that the third combinations have the same laying strategy, are considered more suitable (from the point of time economy).

Fabric is the biggest element of cost in the clothing industry $(50-80 \%)$, so a small saving on fabric with $2 \%$ can significantly affect the pockets of clothing manufacturers [10]. For the purpose of the study, third solutions are the best solutions to reduce the total cost of the order.

Based on the average efficiency and the total fabric consumes, in the third case, we have conducted a better solution than in the first two cases.

Fabric being the major raw material is an important area of cost reduction efforts and Cut Order Plan (Lay Plan) is one of the tools which, if properly used can result in major cost saving [9].

\section{References}

[1] A Puri. 2013. Efficacy of Pattern Making Software in Product Development

[2] Assignmentpoint. Fabric Spreading and Cutting Section of Garments Industry. Retrieved Dec 2014, from Assignmentpoint: http://www.assignmentpoint.com/ science/textile/fabric-spreading-and-cutting-section-ofgarments-industry.html
[3] CHAN SIU HO. 1999. A study of fabric loss during spreading.

[4] David J. Tyler. 2008. Carr and Latham's Technology of Clothing Manufacture. Black well.

[5] Fashion Incubator. What is a marker? Retrieved November 2014, from Fashion Incubator: http://www. fashion-incubator.com/archive/what-is-a-marker/

[6] Gemini CAD SYSTEMS. 2010. Gemini Cut Plan User manual rev X9. Pg 2.

[7] Kahn, Cohen and Soto. 5/18/2005. The Fashion Manufacturing Process A Product Development Approach.

[8] Khush boo Priyambada, Feb 2014. Apparel Production Overview

[9] Kurt Salmon. 2012. Excellence in Global Sourcing.

[10] Mausmi A. 2013. Fabric Utilization, Cut Order Planning.

[11] Ondogan Z, Erdogan C. 2006. The Comparison of the Manual and CAD Systems for Pattern Making, Grading and Marker Making Processes

[12] P Lakshmanakanth. 2012. Spreading and Cutting of Apparel Products.

[13] Prabir Jane. StitchWorld. 2008. Cut Order Planning - The Dot Com Way

[14] Ruth E. Glock. Grance I, Kunz. 2005. Apparel Manufacturing Sewn Product Analysis, Fourth Edition.Pg 395. PEARSON Prentice Hall, New Jersey.

[15] Wong. W, Leung. S.Y. S. 2009. A Hybrid Planning for Improving Fabric Utilization. 